\title{
Imaging C-Fos Gene Expression in Burns Using Lipid Coated Spion Nanoparticles
}

\author{
Aristarchos Papagiannaros ${ }^{1 *}$, Valeria Righi ${ }^{1,2,3^{*}}$, George G. Day ${ }^{2}$, Laurence G. Rahme ${ }^{4,5}$, Philip K. Liu ${ }^{2}$, \\ Alan J. Fischman ${ }^{5}$, Ronald G. Tompkins ${ }^{5}$, A. Aria Tzika ${ }^{1,2,5 \#}$ \\ ${ }^{1}$ NMR Surgical Laboratory, Department of Surgery, Massachusetts General Hospital and Shriners Burns Institute, \\ Harvard Medical School, Boston, USA \\ ${ }^{2}$ Athinoula A. Martinos Center of Biomedical Imaging, Department of Radiology, Massachusetts General Hospital, \\ Boston, USA \\ ${ }^{3}$ Department of Biochemistry “G. Moruzzi”, University of Bologna, Bologna, Italy \\ ${ }^{4}$ Molecular Surgery Laboratory, Department of Surgery, Massachusetts General Hospital and Shriners Burns Institute, \\ Harvard Medical School and Massachusetts General Hospital, Boston, USA \\ ${ }^{5}$ Department of Surgery, Massachusetts General Hospital, Harvard Medical School, Boston, USA \\ Email: \#atzika@hms.harvard.edu
}

Received May 28, 2012; revised July 3, 2012; accepted July 21, 2012

\begin{abstract}
MR imaging of gene transcription is important as it should enable the non-invasive detection of mRNA alterations in disease. A range of MRI methods have been proposed for in vivo molecular imaging of cells based on the use of ultrasmall super-paramagnetic iron oxide (USPIO) nanoparticles and related susceptibility weighted imaging methods. Although immunohistochemistry can robustly differentiate the expression of protein variants, there is currently no direct gene assay technique that is capable of differentiating established to differentiate the induction profiles of c-Fos mRNA in vivo. To visualize the differential FosB gene expression profile in vivo after burn trauma, we developed MR probes that link the $\mathrm{T}^{*}$ contrast agent [superparamagnetic iron oxide nanoparticles (SPION)] with an oligodeoxynucleotide (ODN) sequence complementary to FosB mRNA to visualize endogenous mRNA targets via in vivo hybridization. The presence of this SPION-ODN probe in cells results in localized signal reduction in T2* -weighted MR images, in which the rate of signal reduction $\left(\mathrm{R} 2^{*}\right)$ reflects the regional iron concentration at different stages of amphetamine (AMPH) exposure in living mouse tissue. Our aim was to produce a superior contrast agent that can be administered using systemic as opposed to local administration and which will target and accumulate at sites of burn injury. Specifically, we developed and evaluated a PEGylated lipid coated MR probe with ultra-small super-paramagnetic iron oxide nanoparticles (USPION, a T2 susceptibility agent) coated with cationic fusogenic lipids, used for cell transfection and gene delivery and covalently linked to a phosphorothioate modified oligodeoxynucleotide (sODN) complementary to c-Fos mRNA (SPION-cFos) and used the agent to image mice with leg burns. Our study demonstrated the feasibility of monitoring burn injury using MR imaging of c-Fos transcription in vivo, in a clinically relevant mouse model of burn injury for the first time.
\end{abstract}

Keywords: Positive Contrast; Transverse Relaxation in the Rotating Frame (T2r); Superparamagnetic Iron-Oxide (Uspio); Burn; Skeletal Muscle; C-Fos

\section{Introduction}

The well-known proto-oncogene, c-Fos, encodes a protein that plays key role in every kind of oxidative stress including burn trauma. It has been reported that burns up-regulate the expression of c-Fos within six hours after injury and that this process is mediated through signals originating from the nucleus of monocytes and dermal fibroblasts. This up-regulation is mediated by the effect of protein growth factors that stimulate transcriptional

\footnotetext{
*Aristarchos Papagiannaros and Valeria Righi contribute equally.

\#Corresponding author.
}

activity and increase the delivery of Mrna to the cytoplasm [1-3]. Since c-Fos activation is implicated in numerous crucial intracellular functions such as cell cycle activity and apoptosis, regulation of signal transduction pathways, cellular trafficking, cell proliferation and differentiation, cell survival, and protein folding and proessing and its detection is important in establishing the pathology and path-physiology of burn and the regenerative processes [3]. Detection of c-Fos up to now is done using ex vivo assays following the induction of burn [5], using methodologies such as RT-PCR, Western blot [6] 
or in situ hybridization [1].

Imaging of c-Fos in neural tissues has been reported and it employs ultra-paramagnetic spion agents conjugated to complimentary sODNs. These contrast agents have been employed successfully for magnetic imaging in vivo by local administration to the brain and the use of cell transfection agents as lipofectin [7-9]. Since lpofectin is a generic agent imaging can be performed only after a waiting period and the results obtained following a kinetic study. This imaging method has been limited only to brain tissue since its effectiveness is limited by the transfection capacity of the co-administered agent [7]. Other methods of detection include in vivo microdialysis of the brain using a semi-permeable membrane; a method much more invasive than in vivo imaging [10] but more sensitive than ex vivo electrophoresis [11]. All these methods are invasive to the animal and require local administration of the contrast agent.

Lipid coated nanoparticles have been well established to induce gene transfection in vivo. The application of fusogenic lipids induces fusion of the nanoparticle to the cell membrane allowing the contents of the particle to enter the cells [12]. Fusogenic nanocarriers have recently been proposed as an effective method for delivering a variety of agents; mainly nucleic acids and anticancer drugs [12-14]. This established methodology has achieved the expression of nucleic acids for therapeutic applications in a variety of conditions and when coupled with advanced targeting methodologies offers precision and induced delivery to the desired site [15-17]. The intrancellular fate of the lipids has been long elucidated [18] as well as their ability to target specific pathologic areas in the body, such as inflammation, burn sites or tumors [19]. The intracellular targeting can be adequately precise for targeting specific organelles such as the mitochondria [20, 21].

Lipid coated nanoparticles have also been used for optical imaging in vivo. The lipid coating is changing fundamentally the bio-distribution and the site accumulation of the nanoparticles, leading to self-targeting to the desired site within a few hours. It was demonstrated that similar lipid coated nanoparticles accumulate rapidly to the desired sites and clear form the site with equal speed following their peak concentration at one hour [22,23], while they cannot be detected at four hours following their i.v. administration, a much faster biodistribution compared to the conventional, non-lipid coated solid nanoparticles.

Our aim here was to produce a superior contrast agent that can be administered via systemic injection as oposed to local administration, and will target and accumulate at sites of burn injury. Following the successful accumulation and transfection of the cells by the fusogenic lipid coating, the mRNA expression was imaged and quanti- fied in vivo. Specifically, we developed a pegylated lipid coated MR probe with ultra-small super-paramagnetic iron oxide nanoparticles (USPION, a T2 susceptibility agent) coated with polymer modified fusogenic lipids and covalently linked to a phosphorothioate-modified oligodeoxynucleotide (sODN) complementary to c-Fos mRNA (SPION-c-Fos) and imaged mice with leg burn. Our study demonstrates the feasibility to monitor burn injury using MR imaging of c-Fos transcription in vivo, in a clinically relevant mouse model of burn trauma for the first time.

\subsection{Materials and Methods}

Ultra-small super-paramagnetic iron-oxide nanoparticles (USPIO): Synthetic ultra-small super-paramagnetic ironoxide particles, known generically as Ferumoxtran-10 (USPIO) commercially and as Combidex ${ }^{\circledR}$ in the U.S. (Advanced Magnetics, Cambridge, MA), were used as a molecular-imaging MRI contrast agent. Ferumoxtran-10 nanoparticles are composed of monocrystalline sperparamagnetic iron-oxide magnetite $\left(\mathrm{Fe}_{3} \mathrm{O}_{4}\right)$ cores of about 5 $\mathrm{nm}$ diameter, and they are coated with a dense packing of low molecular weight dextrans to prolong their time in circulation, resulting in a hydrodynamic diameter of about $30 \mathrm{~nm}$.

\subsection{Contrast Agent Preparation}

Contrast agent was prepared by the thin layer evaporation method with slight modifications [22]. Briefly, lipids mixed with the contrast agent (POPC - palmitoyl oleoyl phosphatidyl choline, Cholesterol, DOTAP-2-dioleoyl-3trimethylammonium propane (methyl sulfate salt) and PEG-PE 2000 poly(ethylene glycol)-distearyl phosphoethanolamine (6:3:1:1, $5 \mu \mathrm{g}$ /mouse) were mixed in chloroform $(2 \mathrm{ml})$ with spion $(1 \mu \mathrm{g} /$ mouse $)$ at $30^{\circ} \mathrm{C}$ under vacuum [16]. Following evaporation the lipid coated spions were suspended in saline and kept at rest at $37^{\circ} \mathrm{C}$ for one hour. C-Fos sODN (5'-biotin-labeled antisense sODN: sODN-c-Fos, 5'-catcatggtcgtggtttgggcaaacc-3' [9] was conjugated to the spion conjugated with fluorescein isothiocyanate (FITC) on the 5 terminus and biotin on the 3'-terminus (FITC-sODN-biotin), $255 \mu \mathrm{g}$ of lipid coated spions with 3 pmoles of sODN. Lipid coated spions were incubated for 30 min with c-Fos SODN and were dialyzed against citrate buffer (cut off value 10,000 daltons) for 4 hours twice. The contrast agent was used within 24 hours of preparation.

\subsection{Evaluation of Conjugation}

The conjugates were evaluated using a gel shift assay, as described previously [9]. The conjugated lipid coated sODN spions $(100 \mu \mathrm{l})$ were analyzed using agarose gel 
$(0.8 \%)$ electrophoresis with $3.5 \mu \mathrm{l}$ bromophenol blue solution $(0.25 \%)$ using un-conjugated spions and sODN as standards; $0.26 \mathrm{mg}$ and $765 \mathrm{pmol}$ each. Visualization of the gel was performed using a UV light source and the fluorescence of FITC was used for detection.

\subsection{MRI Experiment}

In vivo imaging was performed in a $4.7 \mathrm{~T}$ horizontal magnet (20 cm bore, Bruker Avance console) equipped with gradient system capable of $39 \mathrm{G} / \mathrm{cm}$, using a custom-built volume coil of $3 \mathrm{~cm}$ inner diameter and $10 \mathrm{~cm}$ active length. Shimming was performed using two repetitions of the automated shimming procedure, followed by manual shimming when necessary (typical water linewidth, $\sim 15-20 \mathrm{~Hz}$ ). We used a clinically relevant mouse model of burn injury [24]. Briefly, six-week old CD-1 mice were anesthetized according to an approved SRAC protocol. The right hind limb of anesthetized animals was subjected to a nonlethal scald injury, representing 3\% - 5\% total body surface area, by immersion in $90^{\circ} \mathrm{C}$ water for $3 \mathrm{~s}$. The image acquisition was performed $12 \mathrm{~h}$ after infusion of SPION.

At the MR scanner, mice were anesthetized with a mixture of isoflurane $(2.0 \%)$ and $\mathrm{O}_{2}(2.0 \mathrm{l} / \mathrm{min})$. Following induction of anesthesia and for the duration of MR imaging, the mice were kept anesthetized with a mixture of isoflurane $(1.5 \%)$ and $\mathrm{O}_{2}(0.6 \mathrm{l} / \mathrm{min})$. The mouse body temperature was maintained at $37^{\circ} \mathrm{C}$ with a warm water blanket.

Anatomical reference images were acquired with RARE (Rapid Acquisition with Refocused Echo) [25] or proton-density weighted FLASH (fast-low angle shot) imaging [26]. A series of FLASH images of constant repetition time (TR) and incremental echo time (TE) for $\mathrm{T}^{*}$ weighting, with typical values $\alpha=35^{\circ}$ (flip angle), were acquired along the axial direction. Acquisition parameters were as follows: $\mathrm{TR}=500 \mathrm{~ms}, \mathrm{TE}=4,6,8,12$, and $14 \mathrm{~ms}, 10$ slices were acquired in the burned region ( $1 \mathrm{~mm}$ thickness, $1.5 \mathrm{~mm}$ gap, $3 \times 3 \mathrm{~cm}$ FOV, $128 \times 128$ matrix size, 8 averages). More details regarding the imaging part are given in our previous investigation [27].

\subsection{Data Analysis}

The image intensity at each voxel was divided by the image noise level. Image noise level was estimated from fitting the histogram of an area in the image background, containing only pure noise voxels, to a Rician distribution. Accordingly, the value of three standard deviations was chosen to threshold-out Rician-noise voxels. An ROI was drawn at the level of the burn area for each mouse, and the total above-threshold signal enclosed in the ROI was computed.

Statistical Analysis: For ROI analysis, we selected MR slices from MR data of each animal group for t-test statistical analysis (two-tailed Student's t-test, $n=6$ per group).

\section{Results}

Figure 1 shows the conjugation of the sODN to spions. SODN and conjugated spions move towards the anode while the non-conjugated spions remained in the well. FTIC was detected by fluorescence with a UV light source $(302 / 365 \mathrm{~nm})$. The spion and the reaction product moved in different directions, due to the high negative charge of the DNA. Also as observed earlier, the conjugation of the SODN with the spion quenches the fluorescence of the FITC. Figure 1 also shows the gel electrophoresis pattern of the ODN, the spion, and the product of the spion SODN reaction in the visible and the UV-vis region.

Figure 2 shows images acquired with different TE (4 and $14 \mathrm{~ms}$ ) in a mouse with burn injury infused with the SPION c-Fos probe. We measured the SNR (snaltonoise ratio) at different echo times ( $\mathrm{T}^{*}$ weighted images Figures 2(a) and (b)) and observed an increase in SNR in images acquired with $\mathrm{TE}=4 \mathrm{~ms}$ versus images acquired with $\mathrm{TE}=14 \mathrm{~ms}$. Interestingly, the SNR in images acquired with $4 \mathrm{~ms}$ in the burn area is more intense compared to the contralateral area (Figure 3), a similar result was observed at $\mathrm{TE}=14 \mathrm{~ms}$ (Figure 4). We thus acquired images with various echo times and to determine the optimal TE for Spion cFos detection. We demonstrated that the optimal TE was $4 \mathrm{~ms}$.

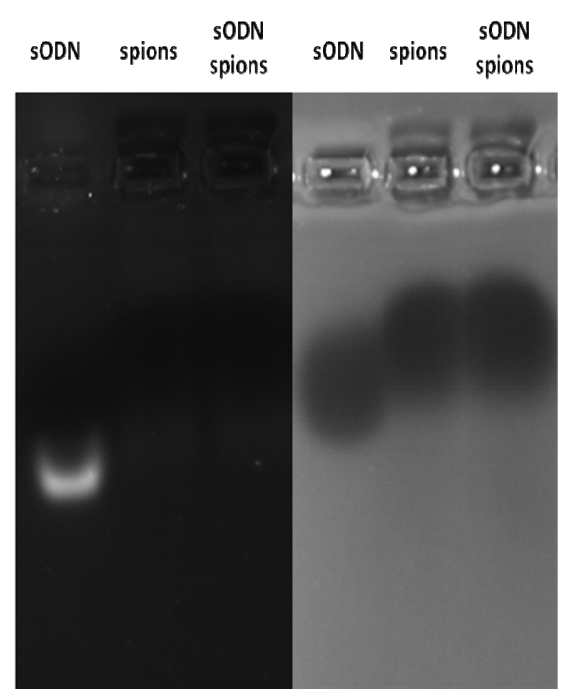

Figure 1. Gel electrophoresis of the ODN, the spion, and the conjugate of the spion sODN (lanes 1 - 3). Images acquired with normal light source or UV lamp (302 - 305 nm), exposure time $8 \mathrm{~h}$. SPION quenches the FTIC fluorescence due to saturation binding but the SODN congugation is obvious from the reversed migration of the spions under the electrical field, due to the negative change of sONA. 
Thus, Figures $\mathbf{3}$ and $\mathbf{4}$ represent the signal to noise detected from the five animals in different echo times, following the injection of the contrast agent. As evident from the figures, there is always higher signal from the burned site compared to that of the control site, following the administration of the contrast agent via the tail vein. Furthermore, Figure 5 represents the detection ability of the contrast agent and the imaging sequence, which demonstrates a statistically significant increase in the signal to noise between the burned site and the control site, at $p<0.05$.

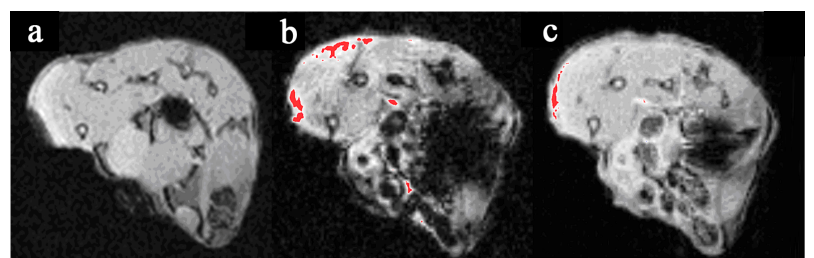

Figure 2. In vivo MR image of a section of the leg of the mouse above the knee showing muscle and bone: (a) anatomical image, after SPION-c-Fos injection, acquired at $\mathrm{TR} / \mathrm{TE}=500 / 4 \mathrm{~ms}$; (b) $\mathrm{TR} / \mathrm{TE}=500 / 14 \mathrm{~ms}$; and (c) are shown.

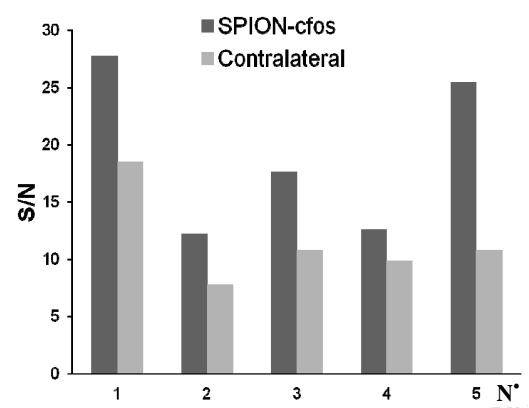

Figure 3. Signal-to-noise ratio $(\mathrm{S} / \mathrm{N})$ in $\mathrm{MR}$ images $\mathrm{T}^{*}$ weighted image: $3 D$ FLASH, $T R=500$, flip angle $=35$ degree) after SPION c-Fos injection at TE $=4 \mathrm{~ms}$ in burn area (labelled with SPION c-fos) and contralateral part. The $x$ axis represents the number of animals $\left(\mathrm{N}^{\circ}\right)$.

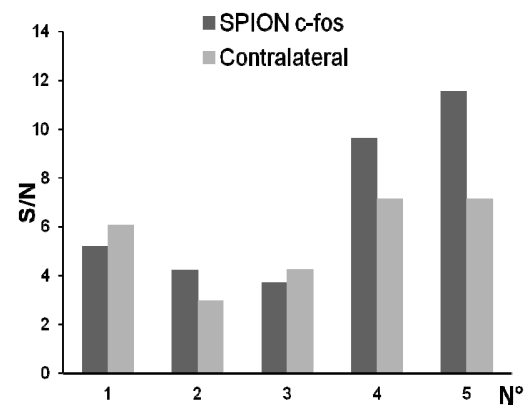

Figure 4. Signal-to-noise ratio $(\mathrm{S} / \mathrm{N})$ in $\mathrm{MR}$ images $\left(\mathrm{T} 2^{*}\right.$ weighted image: $3 D$ FLASH, $T R=500$, flip angle $=35$ degree) after SPION c-Fos injection at TE $=14 \mathrm{~ms}$ in burn area (labelled with SPION c-Fos) and controlateral part. The $\mathrm{x}$-axis represents the number of animals $\left(\mathrm{N}^{\circ}\right)$.

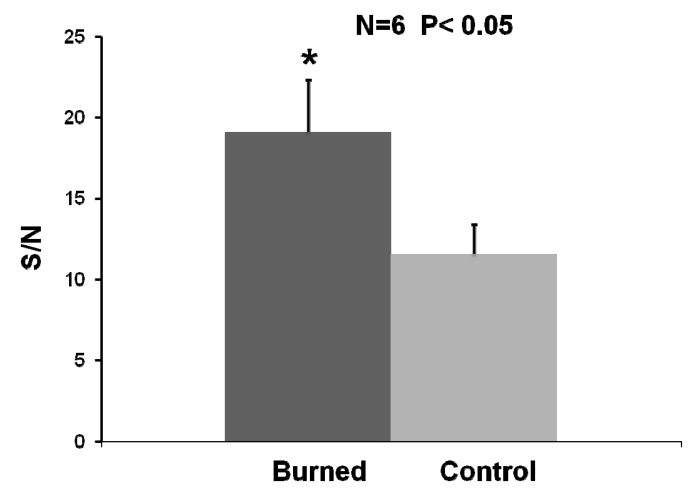

Figure 5. Signal detection in the burned and control groups. Values are means \pm SE, measured within ROIs in burned and contralateral hind limbs. Error bars shown depict standard error of the mean image intensity in the ROI. Asterisk, denotes significant difference between burned and control groups $(n=6$ per group, $P=0.014)$. The $y$-axisrepresents signal-to-noise ratio $(\mathrm{S} / \mathrm{N})$.

\section{Discussion}

Imaging c-Fos expresion in burns offers a unique advantage over other detection methods. It is simple to perform, more reliable and allows for results in real time. Here, we employed a $\mathrm{T} 2{ }^{*}$ weighting imaging method because our agent is a $\mathrm{T} 2{ }^{*}$ susceptibility agent. We used supermaramgentic iron nanoparticles, a $\mathrm{T} 2{ }^{*}$ susceptibility agent, as used by us before [27], which typically produces effective $\mathrm{T} 2{ }^{*}$ relaxation (in gradient echo based sequences) typically an order of magnitude higher for nanoparticles compared to that of Gadolinium complexes. This is partially due to the higher dipolar $\mathrm{R} 2$ relaxivity of these agents, but also due to their much higher magnetization at clinical fields. Furthermore, we produced a superior lipid coated contrast agent, which was self-targeted and accumulated at the burn site and that was administered systemically as opposed to locally. Previous methodologies employed ex vivo observation of the gene expression by western blotting [6] which allowed only indirect assessment of gene activation. The most recent improvement include in situ hybriditzation using bispyrene-modified 2'-O-methyl RNA probes which is useful in the evaluation of ex vivo samples and cultured cells. [28] Using our method we are able to detect transcription of the c-Fos gene in situ and to quantify it with precision using our sensitive magnetic contrast agent which yields high contrast images (Figure 2). Our results suggest for the first time that c-Fos gene expression in burns can be effectively detected non-invasively in vivo and in real time using magnetic resonance imaging.

Our methodology has unique advantages that includeeasy of use and applicability for systemic administration. Previously reported use of similar SPION agents was focused on the gene expression in brain [8,9,29-31] (In 
these studies, specificity was demonstrated using agents with beta-act-in mRNA and random sequence ODNs). In addition the contrast agents had to be administrated locally behind the eye and cell transfection was accomplished using generic agents such as lipofectin and accumulation at the target site is non specific in nature. In fact transfetion is generic and mRNA bound to spions could be imaged only after twelve hours when unbound tracer was released from the cells. Thus, detection of gene expression required a kinetic study of the accumulation and imaging results were correlated to the actual gene expression only after a wash out period. Our methodology is far superior because it allows for transfection at only the pathological site, as only burned tissue attract nanoparticles. Therefore, as evident from the control site, imaging of gene expression took place only at the area of injury.

The motivation behind our work was that c-Fos expression was never imaged in burns, although there are several publications indicating that c-Fos expression can be imaged in cerebral tissue in a variety of pathological conditions. We developed a novel lipid modified contrast agent to label non-invasively the mRNA transcription. Our methodology was to image the gene expression for one day following the administration of the contrast agent in accordance with the protocol established for the gene expression imaging in cerebral tissue and our previous experience with lipid modified contrast agents [7-9, 30,31]. Since we have already established that lipid coated metal nanoparticles accumulate within 2 hours at their target site $[19,22,23]$ and that one day is a sufficient clearance time for non-DNA bond SPIONS, we followed this experimental procedure. Acquisition times were less than 10 min per scan while the retention of contrast agents is published to be several days at their binding site. However, in this study we only performed the experiment at one time point. To this end we have no date on a time series. Nevertheless, based on our data, we may speculate that our procedure will facilitate burn injury monitoring.

Our system tracks and images the gene expression in situ and in real time. As our collaborators have previously indicated $[8,9,31]$ the ability of the contrast agent to track and quantify the gene expression was verified with iron oxide labeling and histology, c-Fos targeting, FITC labelling, and in situ DNA hybridization, as well as comparing the treatment and control sites at the same animals. This system has also been used to trace and track the gene expression for several hours in brain tissue. Following its modification with a self internalizing lipid coating its use can be expanded to a variety of situations and conditions including burn injury, where non invassive system in administration could be advantageous and desired.
Our experimental set-up is designed to demonstrate both the benefits of the contrast agent and that of the imaging sequence. As evident from our results, we constantly observe a higher signal from the burned site than the control and this is also depicted in the cumulative results. This is mainly due to the unique advantages of our positive contrast methodology [27] that enhances the anatomical features and allows for functional molecular imaging of the mouse. Furthermore it is important to notice that the contrast agent is not administered locally but rather systematically, so the differences in the contrast is due to its self-accumulation and self-targeting to the burn site that allows for easy and accurate detection and imaging.

Lipid coated nano-particles accumulate to the target site via passive accumulation with extended permeation and retention effects. Accumulation of our micelle-like formulation at the target site takes place in less than one hour and the remaining time before imaging was required for intracellular accumulation of the agent. We were able to employ the same technique using a different lipid composition to achieve both accumulating at the target site and cell transfection using cationic lipids. This is another advantage of this formulation as it simplifies a multi-step procedure for cell transfection to a single step. Furthermore cationic lipids are highly biocompatible so that once inside the cells, the contrast agent is expected to allow imaging of the mRNA for many hours [8,9]. Our previously reported results indicate that for micellar nano-particles, accumulation at the target site via extended permeation and retention effects takes place within the first hour after administration and clearance is completed within four hours. Since imaging is performed much later, the only conclusion is that the nano-particles have been successfully administered and follow mRNA expression. This conclusion is further substantiated by previous reports that spions administrated with fusogenic agents have a generic cellular transfection pattern which after twelve hours is limited to cells expressing c-Fos as demonstrated with gel electrophoresis $[8,9]$.

Previously, cationic lipids have been mainly used for treatment of pathological conditions by gene delivery and siRNA transfection in vivo [32-35]. In these situations the osDNA is required to remain functional and active during the accumulation time to the desired site. Therefore the cationic lipids are used both for protection of the cargo, targeting to the desired site and accumulation inside the cells. Furthermore our results indicate that the DNA is active since it allows for the detection of gene expression. This contrast agent therefore presents a major improvement in the field of lipid coated solid nano-particles and it can be further developed for imaging gene expression in a variety of human conditions. 


\section{Conclusion}

Our study demonstrates the feasibility of monitoring burn injury using MR imaging of c-Fos transcription in vivo, in a clinically relevant mouse model of burn trauma for the first time. It opens the pathway for MR imaging of the expression of other genes important for monitoring treatments of burn injuries. Our findings represent a new line of research that can support the development of novel therapeutics in the molecular medicine of burn injury. Such therapeutics would have wide-spread applications not only for burn patients, but also for many individuals suffering from other chronic or end stage diseases that exhibit muscle wasting, a condition for which only marginally effective treatments are currently available. Our imminent interest is to perform experiments using novel therapeutic agents for burns and other chronic diseases for monitoring the expression of key regulatory genes such as uncoupling proteins.

\section{Acknowledgements}

This study was supported by Shriner's Hospital for Children research grants (No. 8893) to A. Aria Tzika, and (No. 8892) to Laurence G. Rahme and also in part by a National Institutes of Health (NIH) Center Grant (P50GM 021700) to Ronald G. Tompkins (A. Aria Tzika, Director of the NMR core).

\section{REFERENCES}

[1] X. Fu, et al., "Thermal Injuries Induce Gene Expression of Endogenous C-Fos, C-Myc and bFgf in Burned Tissues," Chinese Medical Journal, Vol. 116, No. 2, 2003, pp. 235-238.

[2] X. Gu, et al., "mRNA and Protein Expression of Transcription Factor C-Fos in Burned Rats and Their Effects on Wound Healing," Chinese Journal of Traumatol, Vol. 3, No. 3, 2000, pp. 141-145.

[3] Q. Guo, Y. Hei and Y. Chen, "The Significance of the Postburn Expression of Proto-Oncogenes C-Fos and CMyc mRNA and Proteins in Rat Myocardial Cells," Chinese Journal of Burns, Vol. 17, No. 1, 2001, pp. 42-45.

[4] A. Medina, et al., "The Role of Stratifin in FibroblastKeratinocyte Interaction," Molecular and Cellular Biochemisrty, Vol. 305, No. 1-2, 2007, pp. 255-264.

[5] J. Chen, J. H. Wang and J. L. Ren, "Changes in the Expression of Apoptotic Genes in the Intestinal Tissue of Scalded Rats before and after Resuscitation," Chinese Journal of Burns, Vol. 21, No. 1, 2005, pp. 55-56.

[6] K. Cho, et al., "CD14- and Toll-Like Receptor 4-Dependent Regulation of C-Fos, C-Jun and C-Jun Phosphorylation in the Adrenal Gland after Burn Injury," Pathobiology, Vol. 71, No. 6, 2004, pp. 302-307. doi:10.1159/000081725

[7] C. H. Liu, et al., "Forebrain Ischemia-Reperfusion Simulating Cardiac Arrest in Mice Induces Edema and DNA
Fragmentation in the Brain," Molecular Imaging, Vol. 6, No. 3, 2007, pp. 156-170.

[8] C. H. Liu, et al., "MR Contrast Probes That Trace Gene Transcripts for Cerebral Ischemia in Live Animals," Official Publication of the Federation of American Societies for Experimental Biology, Vol. 21, No. 11, 2007, pp. 3004-3015. doi:10.1096/fj.07-8203com

[9] C. H. Liu, et al., "Imaging Cerebral Gene Transcripts in Live Animals," Journal of Neuroscience, Vol. 27, No. 3, 2007, pp. 713-722.

doi:10.1523/JNEUROSCI.4660-06.2007

[10] M. L. Oshinsky and J. Luo, "Neurochemistry of Trigeminal Activation in an Animal Model of Migraine," Headache, Vol. 46, No. S1, 2006, pp. S39-S44. doi:10.1111/j.1526-4610.2006.00489.x

[11] J. K. Higa and J. Panee, "Bamboo Extract Reduces Interleukin 6 (IL-6) Overproduction under Lipotoxic Conditions through Inhibiting the Activation of NF-KappaB and AP-1 Pathways," Cytokine, Vol. 55, No. 1, 2011, pp. 18-23. doi:10.1016/j.cyto.2011.02.019

[12] T. Wang, et al., "On the Mechanism of Targeting of Phage Fusion Protein-Modified Nanocarriers: Only the Binding Peptide Sequence Matters," Molecular Pharmacetics, Vol. 8, No. 5, 2011, pp. 1720-1728. doi: $10.1021 / \mathrm{mp} 200080 \mathrm{~h}$

[13] T. Wang, et al., "In Vitro Optimization of Liposomal Nanocarriers Prepared from Breast Tumor Cell Specific Phage Fusion Protein," Journal of Drug Targeting, Vol. 19, No. 8, 2011, pp. 597-605. doi:10.3109/1061186X.2010.550920

[14] T. Wang, et al., "Enhanced Binding and Killing of Target Tumor Cells by Drug-Loaded Liposomes Modified with Tumor-Specific Phage Fusion Coat Protein," Nanomedicine (Lond), Vol. 5, No. 4, 2010, pp. 563-574. doi:10.2217/nnm.10.30

[15] Y. T. Ko, C. Falcao and V. P. Torchilin, "Cationic Liposomes Loaded with Proapoptotic Peptide D-(KLAKLAK) (2) and Bcl-2 Antisense Oligodeoxynucleotide G3139 for Enhanced Anticancer Therapy," Molecular Pharmacetics, Vol. 6, No. 3, 2009, pp. 971-977. doi:10.1021/mp900006h

[16] Y. T. Ko, et al., "Gene Delivery into Ischemic Myocardium by Double-Targeted Lipoplexes with Anti-Myosin Antibody and TAT Peptide," Gene Therapy, Vol. 16, No. 1, 2009, pp. 52-59. doi:10.1038/gt.2008.135

[17] Y. T. Ko, et al., "Self-Assembling Micelle-Like Nanoparticles Based on Phospholipid-Polyethyleneimine Conjugates for Systemic Gene Delivery," Journal of Control Release, Vol. 133, No. 2, 2009, pp. 132-138. doi:10.1016/j.jconrel.2008.09.079

[18] T. Musacchio, et al., "1H NMR Detection of Mobile Lipids as a Marker for Apoptosis: The Case of Anticancer Drug-Loaded Liposomes and Polymeric Micelles," Molecular Pharmacetics, Vol. 6, No. 6, 2009, pp. 1876-1882. doi: $10.1021 / \mathrm{mp} 900164 \mathrm{n}$

[19] A. Papagiannaros, et al., "Near Infrared Planar Tumor Imaging and quantification Using Nanosized Alexa 750Labeled Phospholipid Micelles," International Journal of Nanomedicine, Vol. 4, No. 1, 2009, pp. 123-131. 


\section{doi:10.2147/IJN.S5520}

[20] N. R. Patel, et al., "Mitochondria-Targeted Liposomes Improve the Apoptotic and Cytotoxic Action of Sclareol," Journal of Liposome Research, Vol. 20, No. 3, 2010, pp. 244-249. doi:10.3109/08982100903347931

[21] S. V. Boddapati, et al., "Organelle-Targeted Nanocarriers: Specific Delivery of Liposomal Ceramide to Mitochondria Enhances Its Cytotoxicity in Vitro and in Vivo," Nano Letters, Vol. 8, No. 8, 2008, pp. 2559-2563. doi:10.1021/n1801908y

[22] A. Papagiannaros, et al., "Quantum Dots Encapsulated in Phospholipid Micelles for Imaging and Quantification of Tumors in the Near-Infrared Region," Nanomedicine, Vol. 5, No. 2, 2009, pp. 216-224. doi:10.1016/j.nano.2008.10.001

[23] A. Papagiannaros, et al., "Quantum Dot Loaded Immunomicelles for Tumor Imaging," BMC Medical Imaging, Vol. 10, 2010, 22 p.

[24] J. F. Tomera and J. Martyn, "Systemic Effects of Single Hindlimb Burn Injury on Skeletal Muscle Function and Cyclic Nucleotide Levels in the Murine Model," Burns Including Thermal Injury, Vol. 14, No. 3, 1988, pp. 210219. doi:10.1016/0305-4179(88)90040-X

[25] J. Hennig, A. Nauerth and H. Friedburg, "RARE Imaging: A Fast Imaging Method for Clinical MR," Magnetic Resonance in Medicine, Vol. 3, No. 6, 1986, pp. 823-833. doi:10.1002/mrm.1910030602

[26] J. Frahm, A. Haase and D. Matthaei, "Rapid NMR Imaging of Dynamic Processes Using the FLASH Technique," Magnetic Resonance in Medicine, Vol. 3, No. 2, 1986, pp. 321-327. doi:10.1002/mrm.1910030217

[27] O. C. Andronesi, et al., "Combined off-Resonance Imaging and T2 Relaxation in the Rotating Frame for Positive Contrast MR Imaging of Iinfection in a Murine Burn Model," Journal of Magnetic Resonance Imaging, Vol. 32, No. 5, 2010, pp. 1172-1183. doi:10.1002/jmri.22349

[28] R. Waki, et al., "Development of a System to Sensitively and Specifically Visualize C-Fos mRNA in Living Cells Using Bispyrene-Modified RNA Probes," Chemical Communications, Vol. 47, No. 14, 2011, pp. 4204-4206. doi: $10.1039 / \mathrm{c} 0 \mathrm{cc} 04639 \mathrm{f}$

[29] X. D. Zhang, et al., "Size-Dependent in Vivo Toxicity of PEG-Coated Gold Nanoparticles," International Journal of Nanomedicine, Vol. 6, 2011, pp. 2071-2081.

[30] P. K. Liu, et al., "Transcription MRI: A New View of the Living Brain," Neuroscientist, Vol. 14, No. 5, 2008, pp. 503- 520. doi:10.1177/1073858407309746

[31] C. H. Liu, et al., "Noninvasive Delivery of Gene Targeting Probes to Live Brains for Transcription MRI," Official Publication of the Federation of American Societies for Experimental Biology, Vol. 22, No. 4, 2008, pp. 1193 1203. doi:10.1096/fj.07-9557com

[32] R. R. Sawant, et al., "Polyethyleneimine-Lipid Conjugate-Based pH-Sensitive Micellar Carrier for Gene Delivery," Biomaterials, Vol. 33, No. 15, 2012, pp. 39423951. doi:10.1016/j.biomaterials.2011.11.088

[33] S. Movassaghian, et al., "Dendrosome-Dendriplex inside Liposomes: As a Gene Delivery System," Journal of Drug Targeting, Vol. 19, No. 10, 2012, pp. 925-932. doi:10.3109/1061186X.2011.628396

[34] Y. T. Ko, et al., "Self-Assembling Micelle-Like Nanoparticles Based on Phospholipid-Polyethyleneimine Conjugates for Systemic Gene Delivery," Journal of Controlled Release, Official Journal of the Controlled Release Society, Vol. 133, No. 2, 2009, pp. 132-138. doi:10.1016/j.jconrel.2008.09.079

[35] Y. T. Ko, et al., "Gene Delivery into Ischemic Myocardium by Double-Targeted Lipoplexes with Anti-Myosin Antibody and TAT Peptide," Gene Therapy, Vol. 16, No. 1, 2009, pp. 52-59. doi:10.1038/gt.2008.135 